\title{
Religions, nationalités et discontinuités territoriales. Le cas roumain
} en 1992

Violette Rey, Sophie Vernicos-Papageorgiu

\section{Citer ce document / Cite this document :}

Rey Violette, Vernicos-Papageorgiu Sophie. Religions, nationalités et discontinuités territoriales. Le cas roumain en 1992. In: Espace géographique, tome 23, n4, 1994. pp. 300-311;

doi : https://doi.org/10.3406/spgeo.1994.3331

https://www.persee.fr/doc/spgeo_0046-2497_1994_num_23_4_3331

Fichier pdf généré le 02/04/2018 
Religii, naționalități şi discontinuități teritoriale. Cazul românesc în 1992. - O serie de hârji inédite referitoare la celé 2948 de comune româneçti prezintâ principalele confesiuni (ortodoxă, catolică, protestantă) şi minorităjile (ungară, germană) în Romania anului 1992. Comunităçile minoritare se concentrează pe arii restrănse; comunitatea romăneasca ortodoxă omogenizează teritoriul. Aceste configura^ permit sa se înjeleagâ mai bine cum se perpetueazâ §i cum se reînnoieçte simbioza identitarâ dintre religie §̧i najiune, care este rolul acesteia în individualizarea con§tiin\{elor etnice proprii Europei central-estice, cât de mult este ea un obstacol în calea laicizarii societâjilor. Intensificarea recentâ a construcjiilor de biserici pune în eviden^â noi dimensiuni aie sensibilitâjii confesionale §i permite singularizarea peisajelor religioase régionale.

\author{
Abstract \\ ENG:cultural geography, identity, nationality, religion, Romania, territory
}

\begin{abstract}
Résumé
Une série de cartes inédites des 2948 communes roumaines présente les principales confessions (orthodoxe, catholique, protestante) et minorités (hongroise, allemande) dans la Roumanie de 1992. Les communautés minoritaires se concentrent sur de petites aires; la communauté roumaine orthodoxe homogénéise le territoire. Ces configurations permettent de mieux comprendre comment perdure ou se renouvelle la symbiose identitaire entre religion et nation, quel est son rôle dans l'individualisation des consciences ethniques propre à l'Europe centre-orientale, en quoi elle est un obstacle à la laïcisation des sociétés. L'intensification de la construction des églises fait apparaître de nouvelles sensibilités confessionnelles et permet de singulariser les paysages religieux régionaux.
\end{abstract}




\title{
Religions, nationalités et discontinuités territoriales: \\ le cas roumain en 1992
}

\section{Violette Rey, Sophie Vernicos-Papageorgiou}

\author{
Géophile ENS Fontenay - Saint-Cloud et équipe P.A.R.I.S.
}

\begin{abstract}
RÉSUMÉ- Une série de cartes inédites des 2948 communes roumaines présente les principales confessions (orthodoxe, catholique, protestante) et minorités (hongroise, allemande) dans la Roumanie de 1992. Les communautés minoritaires se concentrent sur de petites aires; la communauté roumaine orthodoxe homogénéise le territoire. Ces configurations permettent de mieux comprendre comment perdure ou se renouvelle la symbiose identitaire entre religion et nation, quel est son rôle dans l'individualisation des consciences ethniques propre à l'Europe centre-orientale, en quoi elle est un obstacle à la laĭcisation des sociétés. L'intensification de la construction des églises fait apparaître de nouvelles sensibilités confessionnelles et permet de singulariser les paysages religieux régionaux.
\end{abstract}

GÉOGRAPHIE CULTURELLE, IDENTITÉ, NATIONALITÉ, RELIGION, ROUMANIE, TERRITOIRE:

REZUMAT.- Religii, naţionalităţi şi discontinuităţi teritoriale. Cazul românesc în 1992. - O serie de hărţi inedite referitoare la cele 2948 de comune româneşti prezintă principalele confesiuni (ortodoxă, catolică, protestantă) şi minorităţile (ungară, germană) în România anului 1992. Comunitătile minoritare se concentrează pe arii restrânse; comunitatea românească ortodoxă omogenizează teritoriul. Aceste configuraţii permit să se înţ̧eagă mai bine cum se perpetuează şi cum se reînnoieşte simbioza identitară dintre religie şi naţiune, care este rolul acesteia în individualizarea conştiinţelor etnice proprii Europei central-estice, cât de mult este ea un obstacol în calea laicizarii societăţilor. Intensificarea recentă a construcţiilor de biserici pune în evidenţă noi dimensiuni ale sensibilităţii confesionale şi permite singularizarea peisajelor religioase regionale.

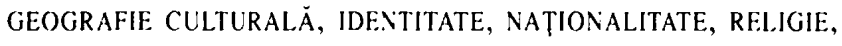
ROMÂNIA, TERITORIU

ABSTRACr.- Religions, nationalities and territorial discontinuity: the case of Romania in 1992. - A serie of unpublished maps of the 2948 Romanian communes shows the distribution of the main religions (orthodox, catholic and protestant) and of minority groups (Hungarians and Germans) in 1992 Romania. Minority communities are concentrated in small areas whereas the Romanian orthodox community acts as the homogenising factor in the whole country. This makes it easier to understand how a symbiotic identity between religion and nation may survive or be revived, what role it may have played in the emergence of the individual ethnic identities typical of Central and Eastern Europe, and to what extent this may constitute an obstacle to the establishment of a non-religious society. That more and more churches are being built is a sign of the emergence of a new religious sense while regional religious landscapes can be identified.

CULTURAL GEOGRAPHY, IDENTITY, NATIONALITY, RELIGION, ROMANIA, TERRITORY 
L'effondrement du système soviétique et la mort de l'idéologie matérialiste que ce système avait tenté de promouvoir ont laissé une béance spirituelle que les églises sont les premières à tenter de remplir car, malgré les persécutions dont elles furent l'objet, leur présence monumentale et leur action plus ou moins souterraine ont traversé la période d'adversité. Mais ce réveil est complexe et ambigu, lourd de tensions autant que d'espoirs, parce qu'est réapparu aussitôt le spectre des antagonismes identitaires, fondé sur le rejeu combiné entre nationalisme et appartenance à une église. La guerre dans l'ex-Yougoslavie s'appuie d'ailleurs en partie sur ce mécanisme. Jacques Attali, dans son tour de l'autre Europe en 1990, note qu'on lui explique qu'«il ne faut pas aider les orthodoxes» (Europe(s), Fayard, 1994, p. 96). C'est là une observation que peut régulièrement faire sur le terrain tout spécialiste de cet espace de contact entre les deux aires chrétiennes. De l'assimilation de «christianisme romain» à «Occident» puis à «Europe», le pas est aisé à franchir. Ce glissement sémantique contemporain, lourd d'enjeux sur la recomposition de l'Europe, retrouve en fait le vieux clivage hérité du Grand Schisme entre Orient et Occident.

Dans l'ambiance générale de transition postcommuniste où s'affirme la remontée des idéologies religieuses et nationalistes, on s'est proposé de localiser sur le territoire roumain les faits religieux et nationaux qui en sont le support, afin de mieux comprendre des situations sociopolitiques complexes et d'identifier les régions à profil religieux particulier. En effet, la Roumanie présente une double individualisation sur la carte ethnicoreligieuse de l'Europe centre-orientale. À l'échelle de la chrétienté européenne, c'est dans sa partie transylvaine que court le contact historique entre les confessions romaine et réformée et la confession orthodoxe, qui va du Monténégro et de l'Albanie du Nord au-delà de Saint-Pétersbourg. Au sein du monde orthodoxe à très forte prépondérance slave, la Roumanie se distingue par sa latinité linguistique et par la présence d'une communauté uniate historique. Comme dans le reste de l'Europe centre-orientale, la cohabitation de plusieurs christianismes se double d'une juxtaposition de plusieurs peuples, Roumains, Hongrois, Allemands (pour les trois communautés historiquement les plus importantes), et inclut aussi une longue expérience d'interférences avec les groupes de religion musulmane. Les deux sources d'énergie spirituelle, dont la langue et la foi sont les vecteurs, se trouvent ainsi en résonance et chargées d'une puissance émotionnelle à la mesure de leur symbiose. Elles soutiennent ici de façon toute particulière la réaffirmation des identités culturelles.

Depuis 1990, le retour à la liberté d'expression et de culte se manifeste de multiples manières. Tout d'abord par diverses expressions de foi religieuse pratiquante, surtout au sein de la jeunesse dont la recherche spirituelle bouscule les institutions ecclésiales. Ensuite par une mobilisation active des églises en vue de reprendre leur rôle d'encadrement pastoral et social, et pour retrouver leurs biens nationalisés. L'expression la plus spectaculaire de cette résurgence religieuse apparaît dans la construction des églises (1). Face à cette effervescence, le nouveau pouvoir politique roumain s'efforce de concilier les différentes pressions politicoreligieuses et de mobiliser leur influence au profit de la reconstruction sociale, avec un esprit de laïcité qui inspirait déjà la constitution de 1923. Mais cet esprit doit être apprécié relativement à la culture orthodoxe dominante, laquelle depuis des siècles a toujours associé d'une manière exceptionnellement symbiotique l'idée nationale et l'appartenance orthodoxe (Thual, 1993).

\section{Questions d'identifés: des chiffres et des lois}

On sait combien la mesure statistique des faits spirituels et des déclarations d'appartenance identitaire est délicate et sujette à caution. À cette imprécise détermination, il serait faux de ne voir que manipulations d'un pouvoir dominant par rapport à des minorités qu'il veut subordonner, même si le phénomène existe en certaines circonstances. Le choix de la déclaration d'appartenance est aussi affaire de comportements individuels. Or, dans certains cas, il est bien difficile de se définir, quand familialement on est issu de parents et grands-parents de nationalités et de religions différentes. Un choix doit être fait, lequel tient compte du contexte politique et économique du moment: faire opportunément jouer la filiation familiale ayant la meilleure

(1) Le mouvement de construction d'églises qui recouvre actuellement l'Europe orientale a commencé en Pologne dès les années 1980, exprimant la résistance spirituelle du peuple catholique polonais à la dictature militaire. 
reconnaissance à l'étranger, ou bien opter pour l'intégration dans le groupe majoritaire (2).

La source d'information est le recensement. En 1930, nationalités et religions ont été recensées, mais dans la configuration territoriale dite de la Grande Roumanie, selon un découpage communal très différent. L'appartenance nationale reste seule notée après-guerre, jusqu'au recensement de 1977. En 1992, illustration du nouvel état d'esprit, nationalités et religions sont à nouveau recensées; malgré la tension propre à ce moment de transition, chacun peut choisir d'affirmer l'appartenance à laquelle il tient. C'est une première exploration de ce recensement qui est ici présentée, pour les 2948 communes rurales et urbaines et les 41 judeţe (départements).

La définition des statuts de citoyenneté et d'association relève de lois, et partout les États font preuve de leur légitime souveraineté à travers ces codifications. Dans la constitution de 1923, le principe de la dissociation entre nationalité et citoyenneté avait été confirmé (3). Il s'agissait là d'un acte de grande portée symbolique en faveur de la construction d'une société à fondement laïque. Dans la loi sur le régime général des cultes (décret $n^{\circ} 177$ du 4 août 1948), prise en

(2) Toute l'histoire sociale de l'Europe centrale est ponctuée par ces glissements, qui sont la preuve de l'identité comme choix de destinée et non seulement comme héritage, et par les problèmes qui s'ensuivent; cf. en particulier le mécanisme de l'intégration des nobles locaux aux aristocraties dominantes, allemande pour les Tchèques, hongroise pour les Roumains de Transylvanie et pour les Slovaques.

(3) Ceci n'est pas automatiquement le cas actuellement dans les pays à forte imprégnation religieuse (cf. Grèce, Israël). D'ailleurs cette dissociation, qui avait nécessité une modification de la constitution de 1866, fut enregistrée sous la pression des grandes puissances au congrès de Berlin en 1878. Cf. DuRandiN C., 1994, Histoire de la nation roumaine, Éditions Complexe, p. 62. La constitution de 1991 déclare la Roumanie «patrie commune et indivisible de tous les citoyens» (art. 4) et elle «garantit aux personnes appartenant aux minorités nationales le droit de conserver, de développer et d'exprimer leur identité ethnique, culturelle, linguistique, religieuse» (art. 6). Par ailleurs, près de $10 \%$ des établissements scolaires primäires et secondaires enseignent dans une langue de minorité pour un effectif d'élèves de $6 \%$ du total des enfants scolarisés. Il s'agit d'un enseignement en langue hongroise à $85 \%$. pleine période stalinienne, le gouvernement supprime de fait la plupart des confessions: 13 sur 60 sont reconnues de façon légale, après acceptation par l'État de leur nouveau statut d'organisation. Les Églises autorisées sont d'une part les «Églises traditionnelles»: orthodoxe, catholique romaine, réformée calviniste, évangélique luthérienne et synodo-presbytérienne, unitarienne, musulmane, mosaiqque, arméno-grégorienne, chrétienne de rite ancien, à l'exception de l'église uniate interdite. Quatre cultes «néoprotestants» s'y ajoutent: baptiste, adventiste du septième jour, pentecôtiste, chrétien selon l'Évangile. Cassant ce carcan, dès fin décembre 1989, le nouveau gouvernement réofficialise l'Église uniate et près de 120 associations religieuses. Il supprime le département des Cultes et lui rend le statut de secrétariat d'État pour les Cultes. Cette libéralisation permet de créer ou de rouvrir des instituts théologiques universitaires et des séminaires pour la formation des cadres religieux de l'enseignement secondaire. Une loi réintroduit le catéchisme dans les écoles élémentaires. Si toutes les confessions en profitent, l'effet de dominance qu'exerce l'Église orthodoxe, dont se réclament $87 \%$ des citoyens, renforce la présence du christianisme oriental et les liens entre son Église et l'État (4).

\section{Degrés de symbiose entre religion et nationalité}

Le fameux cujus regio, ejus religio, qui a tant contribué à simplifier la carte religieuse de l'Europe occidentale en l'adaptant au découpage politique des États à partir de la paix d'Augsbourg (1555), n'a pas fonctionné en Europe centre-orientale. Au contraire, par une combinaison de peuplement peu dense et de structures étatiques peu vigoureuses parce que les grands mouvements migratoires venus d'Asie l'ébranlaient de façon répétée, l'aire centre-orientale de l'Europe est devenue à partir du $\mathrm{XVI}^{\mathrm{e}}$ siècle un lieu de tolérance et de repli des confessions occidentales pourchassées. Ce processus a largement favorisé l'identification simultanée de l'appartenance religieuse et de l'appartenance

(4) En 1993, on dénombre: 4 instituts théologiques orthodoxes nouveaux, à Constanţa, Baia Mare, Craiova, Tărgovişte; la réouverture de l'institut franciscain de Roman, des trois instituts uniates de Cluj, Blaj et Oradea, la réouverture des instituts pentecôtiste et adventiste de Bucarest. Pour les séminaires de niveau lycée: 2 orthodoxes à Tărgovişte et Tulcea; 3 catholiques à Roman, Carei, Bacău; 3 uniates à Oradea, Baia Mare, Cluj; 1 réformé à Aiud; 2 adventistes à Cluj et Craiova. 


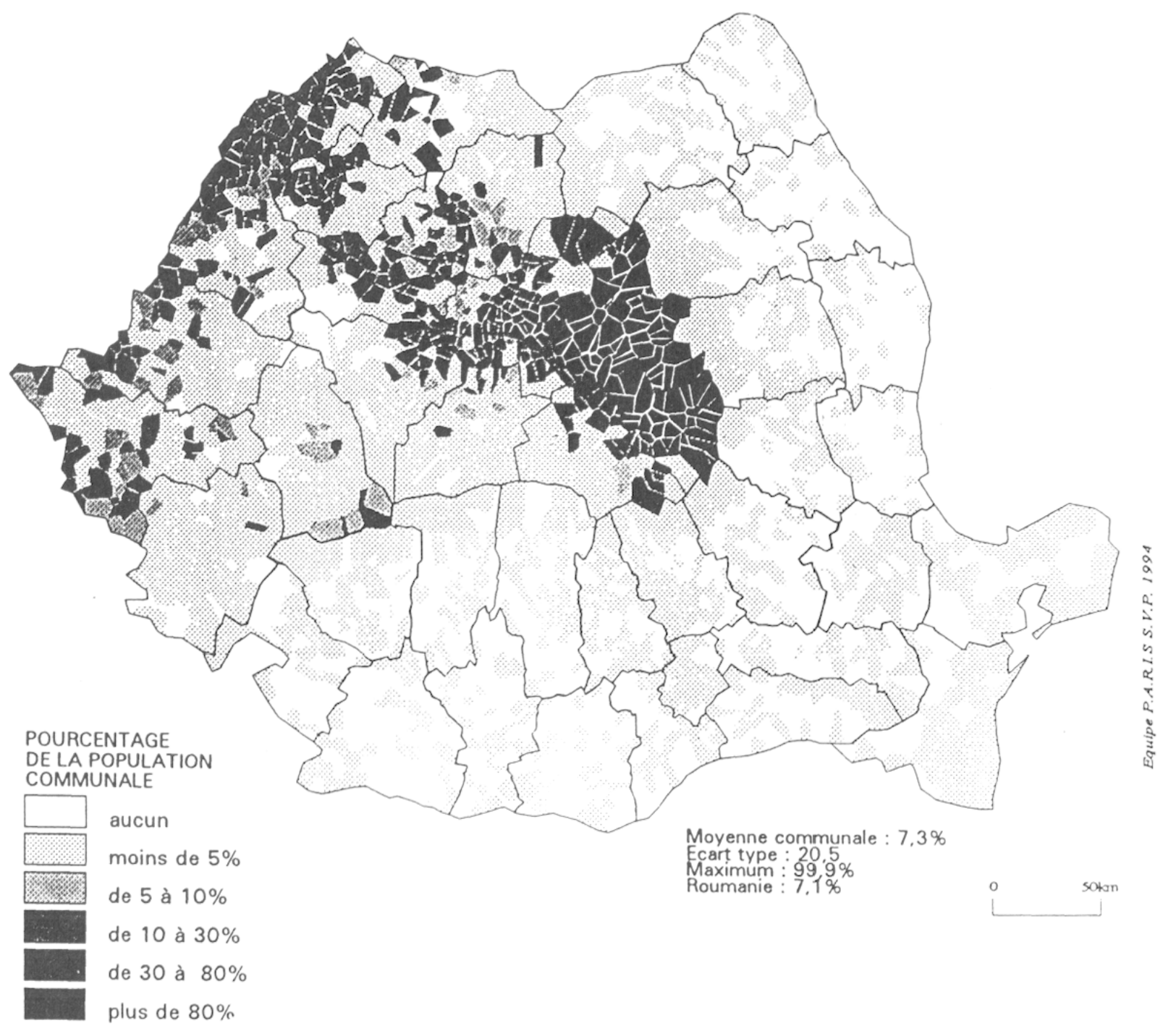

Fig. 1.-Répartition communale de la population de nationalité hongroise en 1992.

ethnique, selon une sorte de cujus religio, ejus natio. Dans le monde chrétien grécobyzantin, un semblable phénomène s'est développé par une conjonction de mécanismes où intervenaient la structure ecclésiale primitive des Églises orientales puis la politique ottomane vis-à-vis des populations non musulmanes sous son influence (5); là aussi, identité religieuse et identité nationale se sont donc le plus souvent confondues.

(5) Dès le concile de Nicée en 325, généralement cité parce qu'il fonde l'autorité particulière de Rome, la prédominance des
Dès lors, il n'est pas étonnant qu'au sein de la Roumanie actuelle on constate une forte corrélation entre les appartenances religieuses et nationales. Évaluée au niveau des 2948 communes, la corrélation linéaire entre le taux

structures synodales est reconnue. avec autonomic des fondations apostoliques; le caractère autocéphale des Églises orientales contemporaines en est issu. I.a politique de l'Empire ottoman, laissant à chaque millet sa liberté de culte moyennant paiement de l'impôt, a dessiné à long terme son rôle à chaque Église d'être la gardienne de la conscience et de l'identité ethnique, puis souvent de devenir un acteur clé pour l'émancipation nationale. 


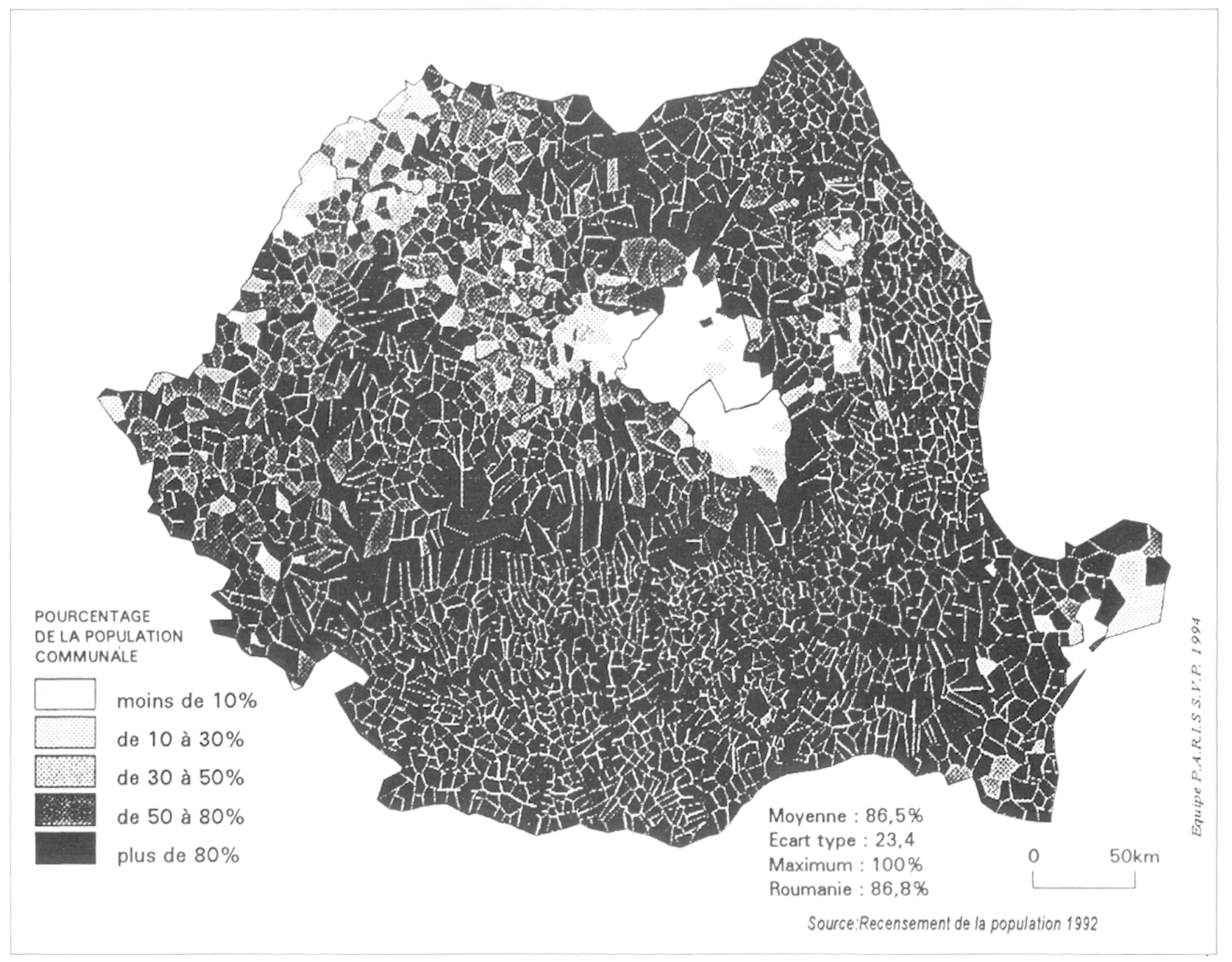

Fig. 2.-Répartition des orthodoxes en 1992.

d'orthodoxes et le taux de Roumains dans la population totale est de 0,88 , entre le taux de protestants et de Hongrois de 0,75 , entre catholiques et Hongrois de 0,56. En fait, ce sont plutôt les corrélations faibles ou absentes qui invitent à réflexion. Dans la sphère strictement religieuse, si les orthodoxes sont en franche opposition avec les catholiques et les protestants $(-0,7)$ et même modestement avec les uniates $(-0,25)(6)$, ce qui compte est que l'implantation catholique et l'implantation protestante ne sont ni associées ni opposées mais seulement indépendantes, indifférentes l'une à l'autre. En raccordant la sphère du

(6) Il s'agit là pour partie d'un effet de boucle, puisque les orthodoxes comptent pour $87 \%$; réunis aux catholiques $(5 \%)$ et aux réformés $(3 \%)$, ils représentent $95 \%$ de l'effectif. religieux aux appartenances nationales, ce qui apparaît est l'absence actuelle de corrélation entre une confession et la minorité allemande: le caractère brutalement résiduel de la communauté allemande, qui comptait encore 359000 individus en 1977 et n'en rassemble plus que 119000 en 1992 , éclaire probablement cette absence de corrélation à propos d'une communauté dont le rôle fut marquant dans l'adoption de la Réforme en Transylvanie au XVI ${ }^{\mathfrak{e}}$ siècle (Hontérus à Braşov) et dont l'apport souabe dans le Banat au $\mathrm{XVIII} \mathfrak{I}^{\mathfrak{B}}$ siècle fut principalement catholique.

Trois autres situations méritent attention. Le cas des Tziganes est original. Leur effectif en 1992 (409 000 habitants, $1,8 \%)$ et leur taux de croissance depuis 1977 (180\%, 227000 personnes) les placent au troisième rang des communautés. Il a cependant surpris par sa modestie. Cette 


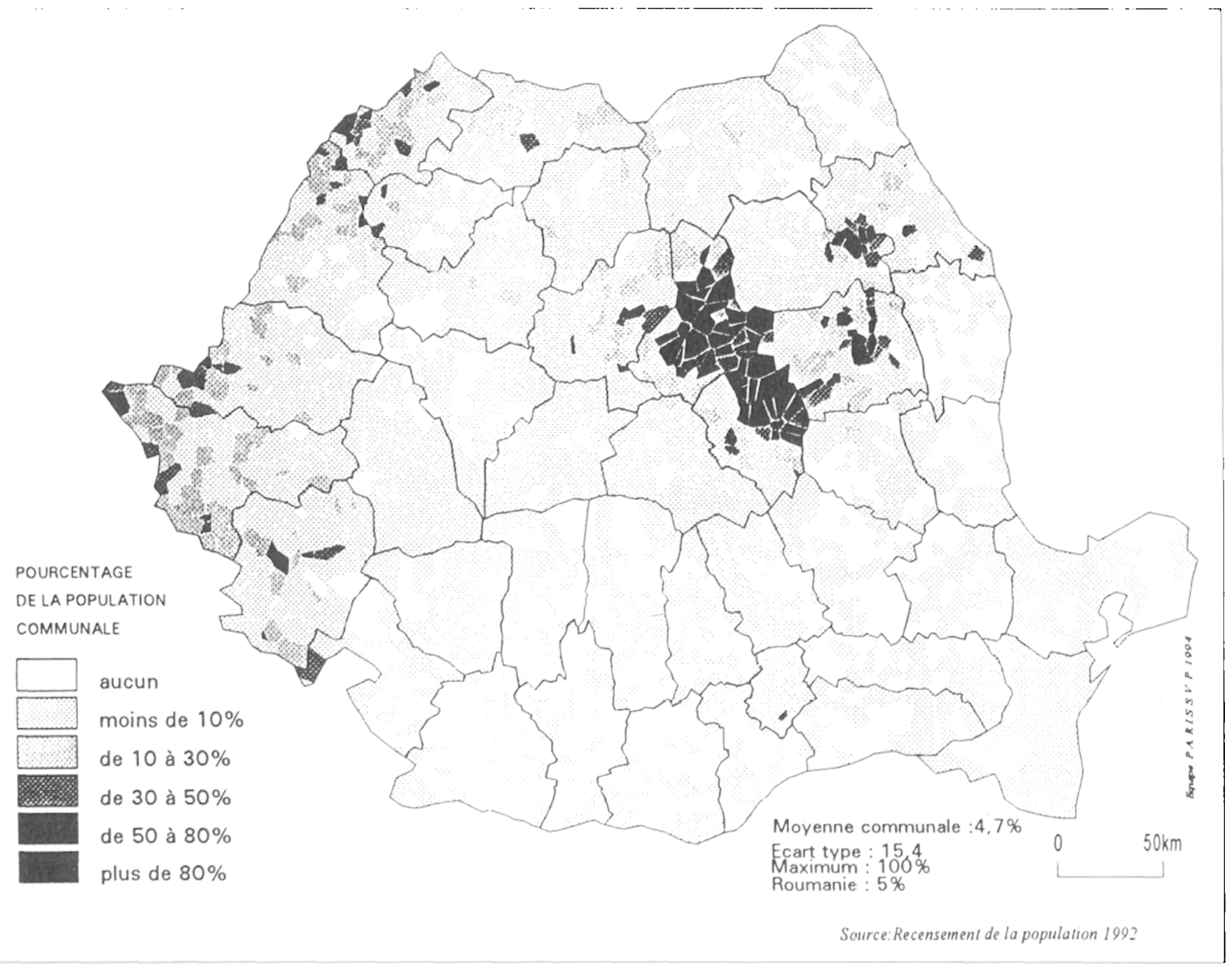

Fig. 3.-Répartition des catholiques en 1992.

sous-représentation, comparativement à la réalitć sociologique de la présence tzigane, est également observée par les statisticiens des pays voisins (Slovaquie, Hongrie, Bulgaric). Deux interprétations peuvent être proposées: l'une est que les Tziganes choisissent l'intégration et se déclarent membres de la nation au sein de laquelle ils vivent; l'autre tient que l'unité tzigane est partiellement un effet d'amalgame, créé par le regard des autres. Géographiquement peu concentrés, en faible corrélation négative avec la population roumaine et orthodoxe $(r=-0,3)$ et en faible corrélation positive avec la population hongroise $(r=0,2)$, ces mesures laissent supposer que les Tziganes orthodoxes se sont déclarés Roumains (7).

À l'inverse, la communauté religieuse musulmane représente une minorité de 56000 personnes. Elle est surtout composćc dc Tatars (25 000) ct de Turcs (29 000); le petit groupe turc est en progression par rapport à 1977 (23 000) et se concentre en Dobroudja. La communauté juive, très présente en Moldavie avant-guerre, a presque totalement disparu (9 100 personnes contre 24600 en 1977). Les catégories «athée» et «sans religion déclarée», qui rompent totalement avec toute association à une nationalité particulière et qui avaient été encouragées par le système socialiste, ne comptent guère en Roumanie: 36000 «athées", 15000 «sans religion déclarée», c'est à peine $0,3 \%$ de la population totale. Ces catégories apparaissent comme

(7) Ceci expliquerait aussi pourquoi les fortes communautés tziganes du piémont olténien sont invisibles dans le recensement. 


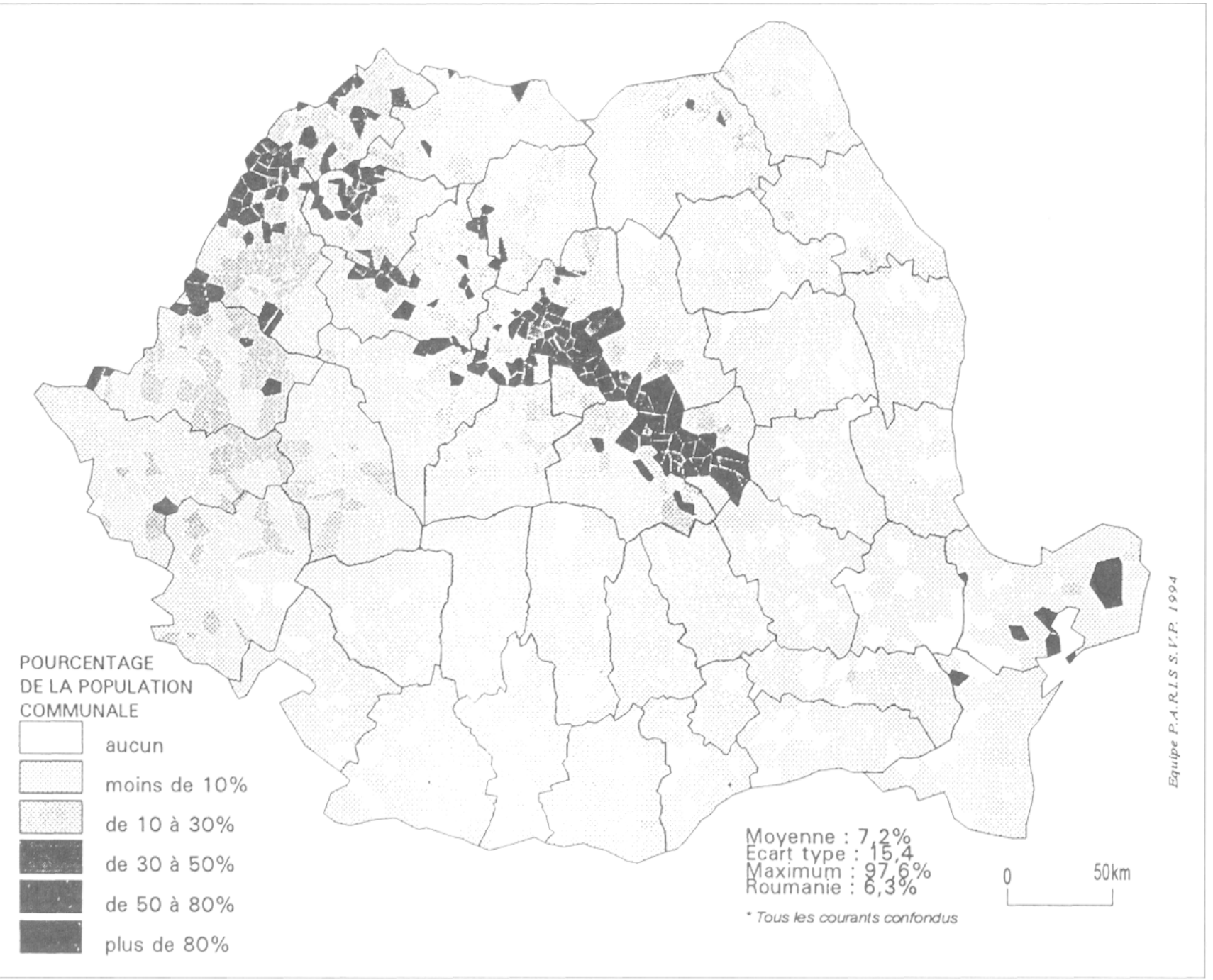

Fig. 4.-Répartition des protestants en 1992.

étrangères aux valeurs du pays et n'existent d'ailleurs à peu près que dans les villes $(78 \%)(8)$

\section{Enracinements ef diffusions}

C'est bien dans la géographie même du double phénomène, religieux et national, que git une part essentielle des problèmes épisodiques de cohabitation culturelle et de tension géopolitique. Si établir cette géographie n'est certes pas une simple

(8) À comparer avec les $40 \%$ d'athées en République tchèque et les 10\% en Slovaquie. au recensement de 199i. Cf. Davik P., «Éléments de la différenciation culturelle et politique du territoire tchèque», in RFy V.. La Tihé(or-Slovaquie en 1992, Éditions de l'ENS, 1994. affaire de cartes, celles-ci sont précieuses pour clarifier les débats et dresser le paysage confessionnel. Le choix du niveau cartographique pour en rendre compte est d'une grande importance, comme le rappelle la simple comparaison des coefficients de corrélation selon qu'ils sont établis au niveau communal ou départemental: entre protestants et catholiques, les coefficients sont de 0,57 au niveau départemental, mais seulement 0,07 au niveau communal; entre orthodoxes et Roumains, de 0,98 et 0,88 respectivement.

De telles différences de valeurs de corrélation selon le niveau d'agrégation donnent le sens des interprétations géographiques à établir. L'échelon départemental, porteur des corrélations les plus fortes, est significatif pour une approche des ambiances régionales: une individualisation de la Transylvanie apparaît ainsi clairement, délimitée par la ligne historique 


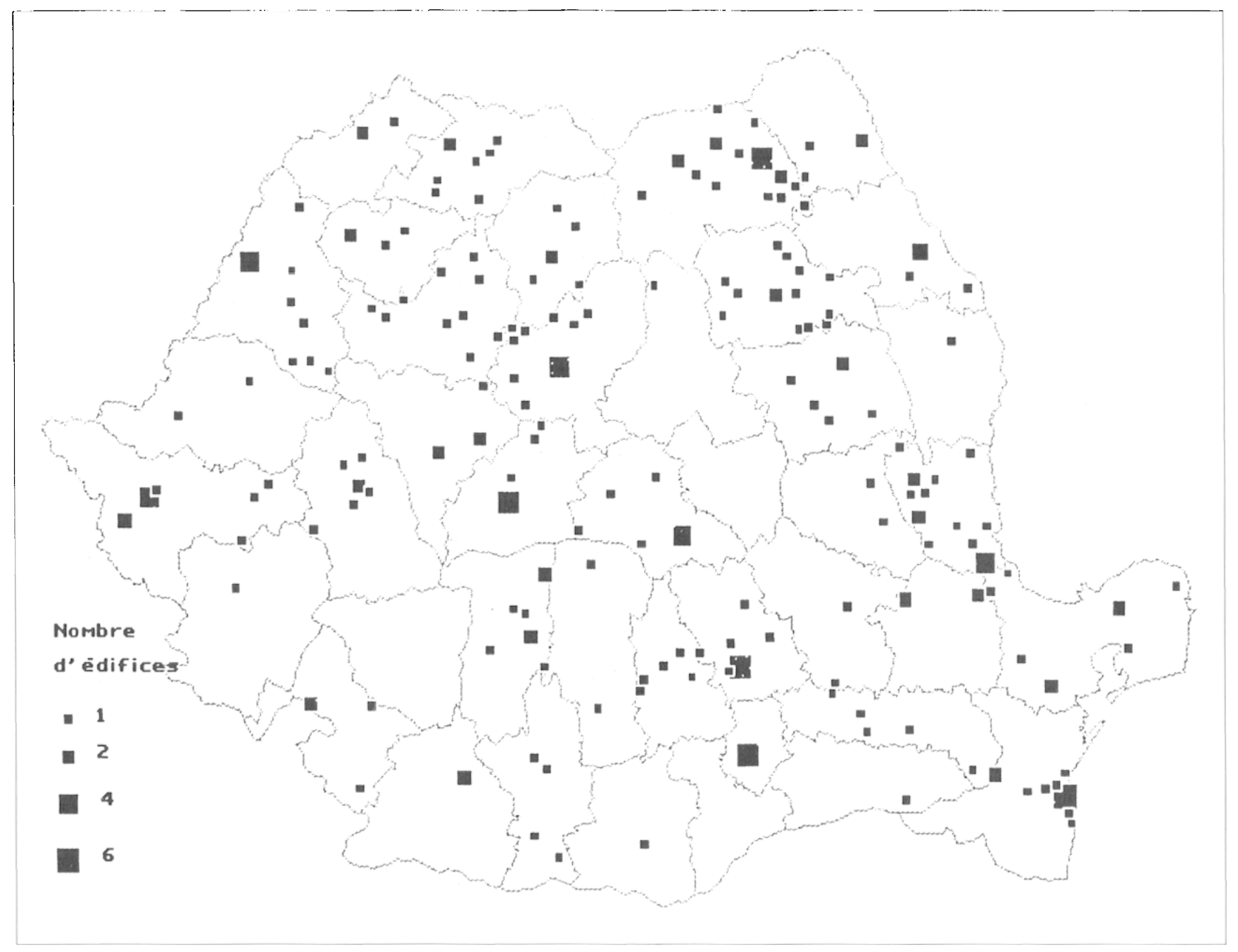

Fig. 5.-Nouvelles églises orthodoxes en 1992.

de la frontière qui, pendant des siècles, a séparé le royaume de Hongrie puis l'empire d'Autriche-Hongrie des principautés roumaines. Cette individualisation, produite par la diversité des confessions et des nationalités présentes, s'oppose à la forte homogénéité roumaine et orthodoxe de toute la partie moldo-valaque du territoire; elle était bien plus marquée encore aux débuts des années 1950, si l'on prend en considération la proportion des nationalités non roumaines.

Par contre, l'échelon départemental est sans réelle portée pour les communautés élémentaires puisque, au niveau communal, elles sont le plus souvent majoritaires dans l'une ou l'autrc des confessions, voire exclusives des autres. Ainsi, dans l'aire hongroise, pourtant fort continue en Transylvanie centrale, les communes sont catholiques ou protestantes, rarement mixtes. Cette disposition spatiale en cellules juxta- posées, vivante en 1992, permet de comprendrc comment se reproduisent sur la longue durée les mécanismes d'individualisation de petites communautés, au sein desquelles s'invente et se conserve un ensemble d'attitudes, de valeurs, de coutumes qui constituent la conscience ethnique, si spécifique de l'Europe centrale. Cette description met également en relief la distinction entre l'espace à dominante roumaine orthodoxe et celui dit de la "Transylvanie historique»: la ligne de crêtes des Carpates ne sépare pas, la roumanité orthodoxe a la même présence en Maramures qu'en Moldavie, dans les monts Apuseni et de Hunedoara qu'en Olténie.

Les cartes des minorités religieuses montrent des formes d'extrême concentration géographique, avec deux types de foyers. Les confessions protestantes dessinent une diagonale SE-NO du pays sicule de Covasna au Bihor, prolongée 


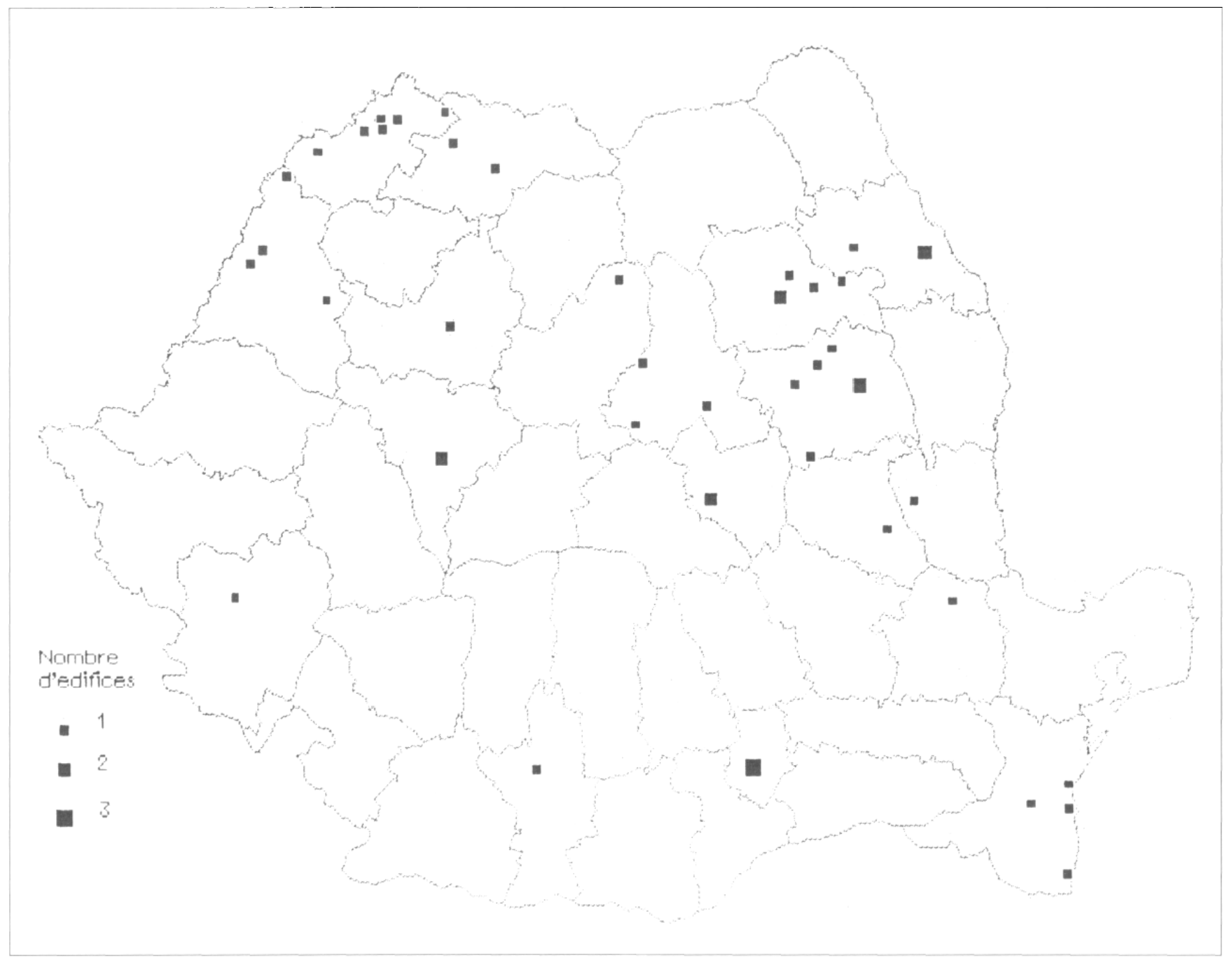

Fig. 6.- Nouvelles églises catholiques en 1992.

d'une bande en bordure de la frontière hongroise, traversant les régions à peuplement hongrois sans vraiment toucher le Banat. La confession catholique se rapproche de cette configuration, mais en décalé et plutôt sous la forme de noyaux: Banat, bloc de Harghita-Covasna, et petits noyaux ceangai et moldaves dans la vallée du Siret autour de Roman et Bacău. Quant à la confession uniate, elle paraît en léger voile continu sur toute la Transylvanie.

À ces enracinements et à ces discontinuités qui reflètent toute l'histoire du peuplement sur ce territoire, on peut ajouter des signes d'une dynamique religieuse toute récente, lue à travers les demandes de construction ou de réparation des édifices religieux (9). Contrairement à l'idée largement répandue en Occident à la fin du régime Ceaucescu, lors de la grande peur provoquée par les regroupements villageois, les démolitions d'églises ont été limitées, et plutôt associées à l'éventrement des vieux centres-villes, au nom de la modernisation urbanistique, en particulier à Bucarest. Une vague antérieure correspondit à la collectivisation des campagnes, qui a aplani et nettoyé les champs de certains hameaux de tout édifice symbolique, afin de créer un parcellaire géant, ouvert aux machines. De plus, le patrimoine reli-

(9) La liste des édifices à caractère religicux ayant fait l'objet d'une demande de subvention a été relevée auprès des registres du ministère des Cultes pour 1990, 1991, 1992; toute construction n'ayant pas fait l'objet d'une demande n'est pas prise en compte; toute demande n'est pas synonyme de mise en chantier. Sur le terrain, il semble que les édifices orthodoxes soient les mieux répertoriés sur ces listes. 


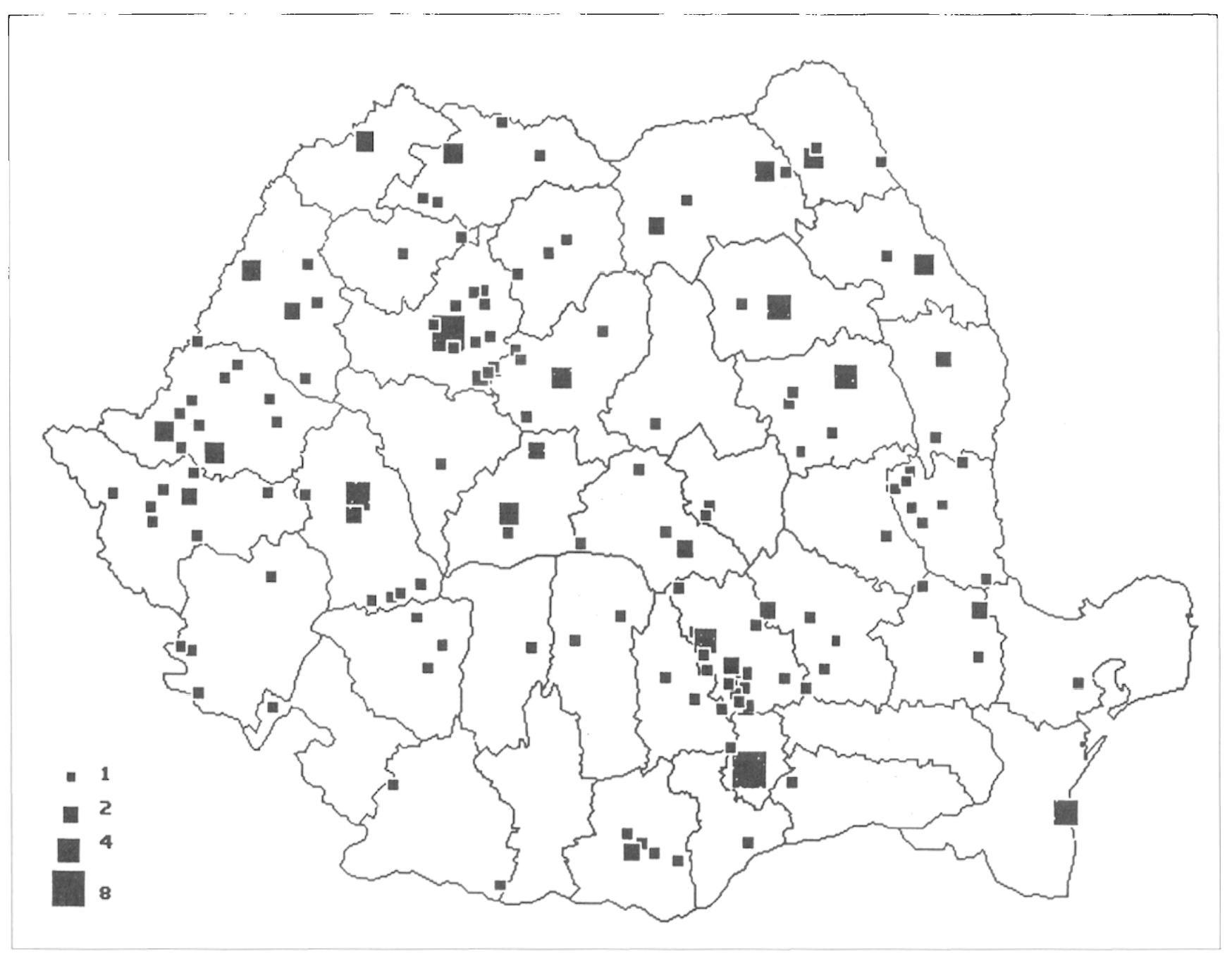

Fig. 7._Nouvelles églises protestantes en 1992.

gieux est mieux entretcnu cn Roumanie que dans les pays ex-socialistes voisins, parce que la pratique religieuse était restée plus libre et plus assidue.

Malgré la déclaration d'appartenance religieuse, qui s'élève à $87 \%$ pour les orthodoxes, ou malgré l'image d'une quasiomniprésence orthodoxe que peut laisser une observation rapide de terrain, l'Église orthodoxe ne participe qu'à hauteur de $45 \%$ à ces dossiers de demande; en revanche, les Églises catholique et protestantes sont très au-dessus de leur rang. Il est certes normal que certains cultes en début d'implantation fassent preuve d'un effort particulier de construction et qu'au contraire la confession la plus répandue et enracinée n'ait pas ce même besoin; toutefois, ce si grand déséquilibre révèle l'inégale capacité financière des croyants qui sont à l'origine de ces dossiers, et sans doute aussi la différence de l'assise économique des communautés auxquelles ils appartiennent.

Les demandes de construction et de réparation d'églises orthodoxes couvrent tout le territoire, à l'image de la présence orthodoxe. Elles concernent surtout les villes et les communes suburbaines, où la croissance démographique a été très forte et ne s'est pas accompagnée de la construction d'édifices du culte. Les demandes de construction d'églises protestantes présentent la géographie la plus originale: au-delà de leurs foyers initiaux tenus par l'Église réformée sur l'axe de Satu Mare à Covasna, elles essaiment en direction de l'Est; les églises des cultes néoprotestants apparaissent partout: autour de Cluj, autour d'Arad et Timişoara en Banat; elles sont nombreuses en Munténie et s'implantent dans les villes importantes de Moldavie; 


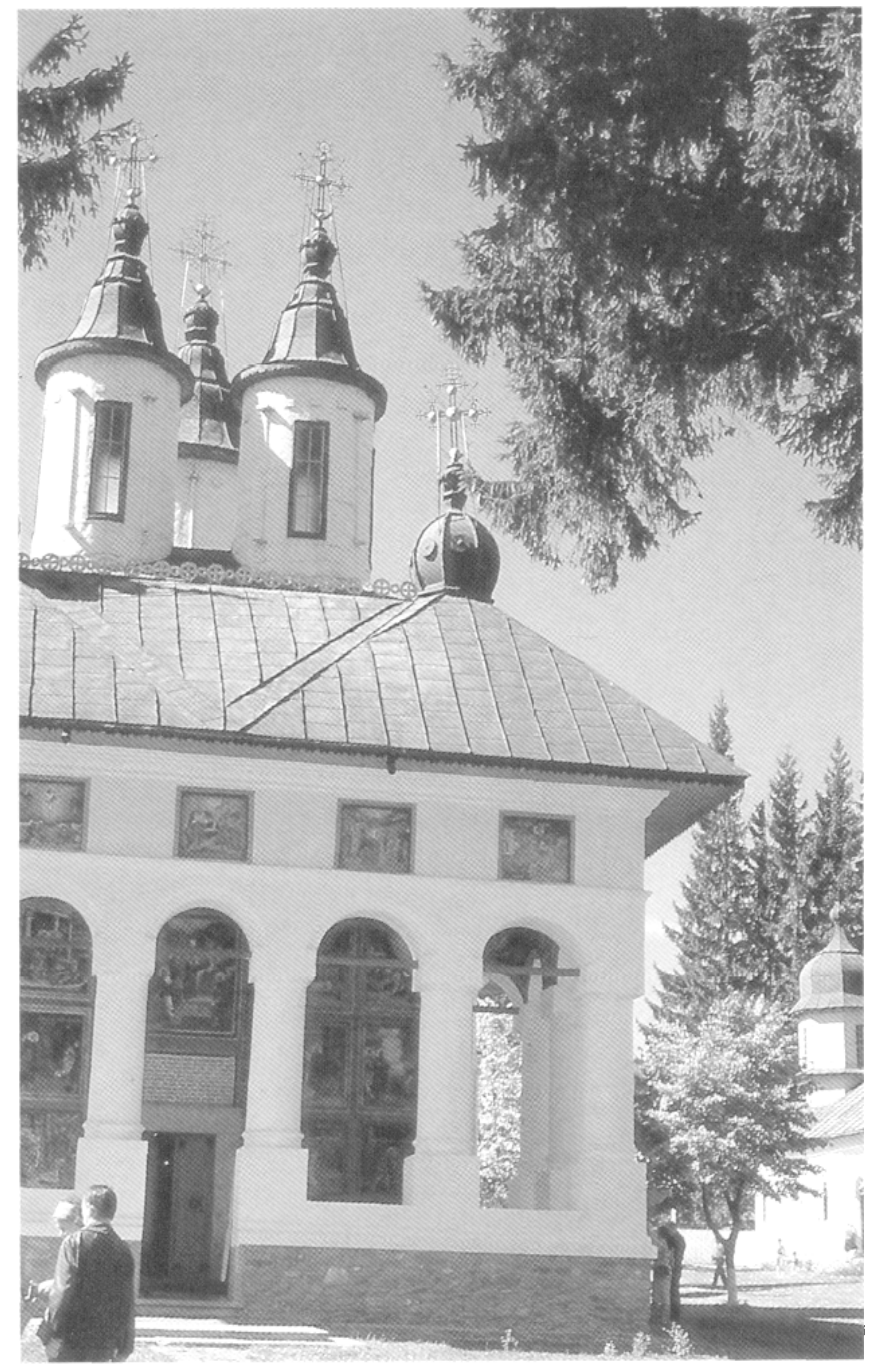

Phot. 1.- Église orthodoxe dans la cours d'un monastère, en Moldavie (cliché 1994).

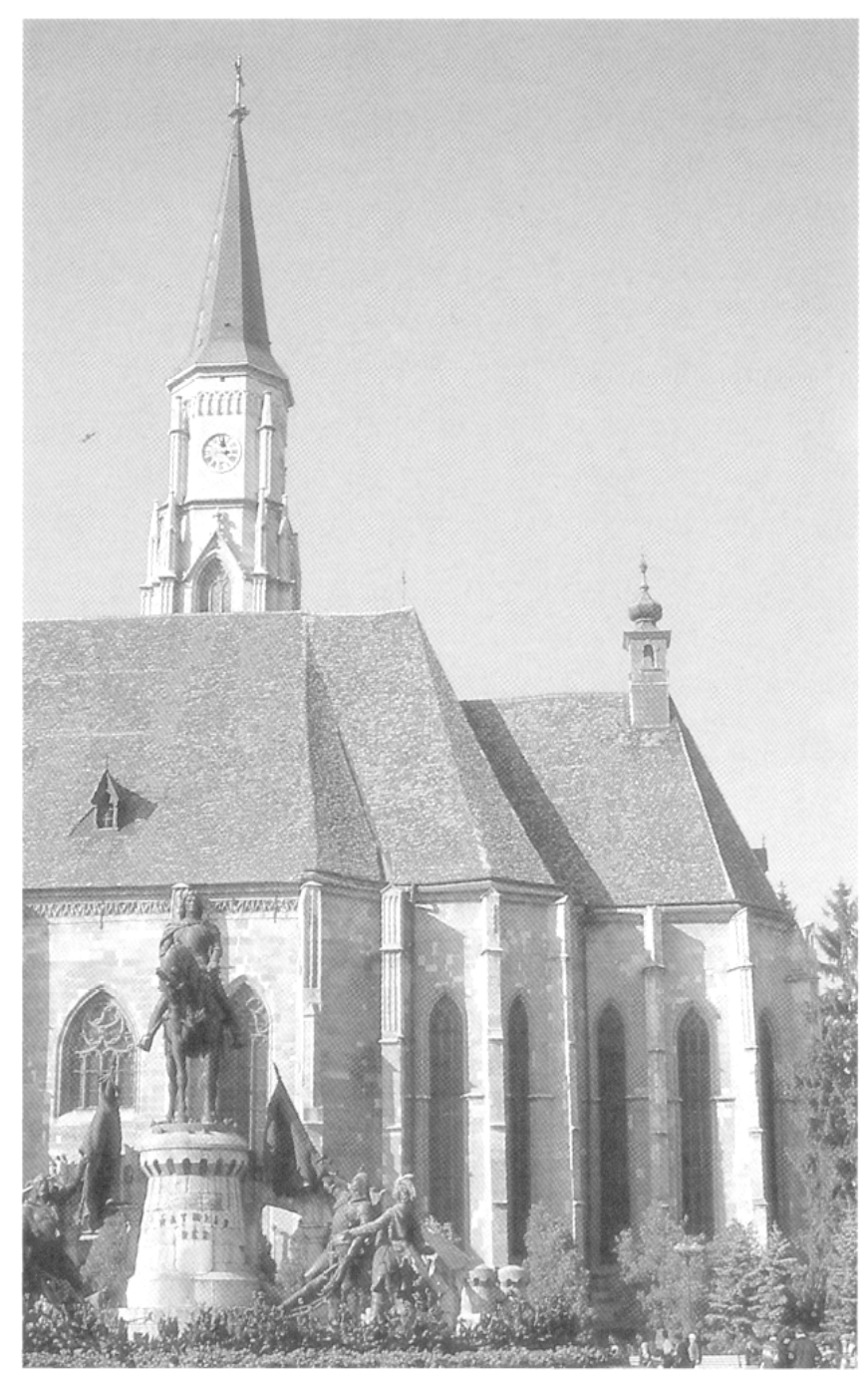

Phot. 2.- Cathédrale gothique catholique Saint-Étienne de Cluj avec la statue équestre de Mathias, roi de Hongrie (1440-1490). (Cliché 1991). Aujourd' hui, une église orthodoxe est en cours de construction à proximité.

Tout à fait intéressante en est la cartographie détaillée, confession par confession (pentecôtiste, baptiste, adventiste): chaque culte a sa géographie particulière, dont il reste à interpréter les origines. L'investigation visera en particulier à apprécier dans quelle mesure ce phénomène d'adhésions néoprotestantes s'implante là où, antérieurement, d'autres sectes dissidentes avaient existé, là où il révèle d'anciennes cohabitations ethniques plus ou moins oubliées. Les nouvelles églises catholiques ne touchent pas le principal noyau catholique du pays, dans la région de peuplement hongrois de Harghita-Covasna, où l'entretien des bâtiments fut toujours maintenu et où le besoin en édifices nouveaux n'existe guère, compte tenu du réseau 
antérieur. Une diffusion sur l'aire orthodoxe apparaît clairement à partir d'autres noyaux catholiques antérieurs; ainsi en est-il en Moldavie centrale autour de Bacău et de Roman ou dans l'extrême Nord-Ouest autour du foyer catholique de Satu Mare. Enfin, le nombre dérisoire de demandes en faveur d'édifices uniates ( 5 dont 3 en Satu Mare) révèle la crise de cette Église grécocatholique, dont l'existence a toujours été placée sous le signe de la tension et du conflit politique. De liturgie byzantine avec un rattachement canonique au Saint-Siège de Rome, elle remonte au concile de Florence (1439); ses débuts sont contemporains des mouvements de la Réforme, et son implantation en Transylvanie commence au XVIII ${ }^{\mathfrak{e}}$ siècle dans la foulée du renforcement de l'emprise autrichienne. Trop souvent considérée comme le résultat d'une simple manipulation de l'Église de Rome et du mouvement de la Contre-Réforme, bête noire de l'Église orthodoxe (10), cette Église eut un très grand rôle historique dans la redécouverte de la romanité des Roumains, de la latinité de leur langue et dans le développement au XIX ${ }^{\mathrm{C}}$ siècle d'une conscience nationale fondée sur cette image identitaire; mais elle a été la plus touchée par le stalinisme, supprimée et rattachée d'office à l'Église orthodoxe en 1948. Dans la Transylvanie de l'entre-deux-guerres, la proportion des uniates atteignait presque $30 \%$ (recensement de 1930); leur discrétion actuelle est probablement à rapprocher du contexte ethnique particulier à la Transylvanie; il semble qu'après 40 ans de pratiques cultuelles tenues dans les églises orthodoxes, et afin d'éviter une possible assimilation à la communauté magyare, les Roumains de tradition uniate soient dans leur majorité restés dans le giron de l'orthodoxie. La question n'est cependant pas close, et des problèmes de restitution de biens fonciers font surface.

Cette première analyse du paysage religieux en territoire roumain a précisé les physionomies régionales typées et les tendances actuelles. Tandis que l'Olténie s'affirme

(10) L'Église orthodoxe de Transylvanie n'était que tolérée, à la différence des autres confessions catholique, luthérienne. calviniste et unitarienne; par un décret de 1692, prêtres et fidèles orthodoxes qui passaient à l" "union" ćtaient assurés des mêmes avantages que les autres chrétiens, ce qui améliorait considérablement leur statut politique. comme la plus homogène religieusement et nationalement, la Transylvanie et le Banat-Crisana, au contraire, conservent une image plurielle combinant des noyaux dispersés et des discontinuités locales nombreuses. La Moldavie, très imprégnée de culture orthodoxe roumaine, révèle aussi une diversité potentielle, reflet de son ouverture ancienne aux influences culturelles lointaines. Le mécanisme général de rétrécissement de l'éventail des nationalités et de réduction du poids des confessions minoritaires par rapport à la confession orthodoxe dominante fonctionne, comme en d'autres époques dans toute l'Europe occidentale. Mais il fonctionne selon la modalité d'une symbiose identitaire, conservée entre les deux piliers de la religion et de la nationalité, ce qui ne favorise pas l'émergence de la laïcitć. L'ignorance ou le rejet de l'athéisme et le déclin uniate sont à comprendre par le sentiment de danger que courent les communautés en cette période de grande crise politique. Ils expriment un réflexe de conservation d'identité. Si l'accueil réservé aux nouveaux cultes dépend en partie de l'attrait qu'exerce une vision eschatologique en provenance de pays occidentaux, il témoigne aussi d'une attente spirituelle que ne sait pas remplir le modèle agnostique défaillant.

\section{Références}

Code législatif et institutionnel pour les minorités nationales en Roumanie (1994). Bucarest: Institut roumain des Droits de l'Homme.

IACOB G. (1994). «L'orthodoxic sur le territoire roumain». Communication au Semi-centenaire de l'Institut de géographie de l'Académie roumaine, à paraître.

Iorga N. (1992). Byance après Byzance. Paris: Balland, 312 p.

Köpecza B. dir., (1992). Histoire de la Transylvanie. Budapest: Académie des Sciences, $740 \mathrm{p}$.

KriNdatch A.D., VÉdDÉNINE J.A. (1993). «L'évolution du paysage confessionnel de la Russie et des pays voisins». L'Espace géographique, XXI, n 1, p. 69-75.

Mihaescl: H. (1993). La Romanité dans le Sud-Est de l'Europe. Bucarest: Académie roumaine, 600$) \mathrm{p}$.

Roberti J.-C. (1992). Les Uniates. Paris: Éditions du Cerf, I30 p.

Thlal. F. (1993). Géopolitique de l'orthodorie. Paris: Dunod, $125 \mathrm{p}$. 